\title{
The effect of lowering salt intake on blood pressure and biochemical indices of cardiovascular and bone health in adult subjects with slightly elevated blood pressure
}

\author{
F. Leenhardt ${ }^{1}$, E. Arendt ${ }^{1}$, J. Kerry ${ }^{1}$, S. Kenny ${ }^{1}$, J. Murnane ${ }^{1}$ and K. D. Cashman ${ }^{1,2}$ \\ ${ }^{1}$ School of Food and Nutritional Sciences, University College Cork, Ireland and ${ }^{2}$ Department of Medicine, \\ University College Cork, Ireland
}

There is evidence that relatively modest restrictions in salt intake have the potential to produce a significant fall in average blood pressure (BP) at a population level with a concomitant substantial impact on the burden of morbidity and mortality from CVD ${ }^{(1)}$. Reducing salt intake may also impact positively on skeletal metabolism, as a high salt diet increases urinary Ca loss and bone turnover ${ }^{(2)}$. Two food groups (meat and fish (especially processed meats) and bread) account for over $50 \%$ of salt intake from foods in Ireland, with the remainder contributed by various other processed foods ${ }^{(3)}$. The aim of this study was to examine the effect of dietary salt restriction on BP and biochemical markers of bone turnover in free-living adults with slightly elevated BP (stage 1 hypertension). Low-salt bread $(0.3 \mathrm{~g} / 100 \mathrm{~g})^{(4)}$, unsalted spread and reduced salt luncheon meats formed key elements of the dietary salt restriction approach.

Ninety-six Caucasian men and women (mean age, 46.7 years) with a resting systolic BP $\geq 120$ and $<160 \mathrm{mmHg}$ and/or a diastolic BP $\geq 80$ and $<95 \mathrm{mmHg}$ (who were not taking any anti-hypertensive medication) were recruited and randomly assigned to follow either the reduced-salt diet or their usual diet (control) for 5 weeks and then cross-over to the alternate dietary regimen for a further 5 weeks. Systolic and diastolic BP was measured at baseline and endpoints with the OMRON 705-IT automated BP monitor. Urinary Na was measured in $24 \mathrm{~h}$ urine collections. The completeness of the $24 \mathrm{~h}$ urines was assessed by creatinine and only those adjudged complete were used in the statistical analysis. Osteocalcin, bone-specific alkaline phosphatase, C-terminal telopeptide of type 1 collagen (CTx) and parathyroid hormone (PTH) were analysed in serum and N-terminal telopeptide of type 1 collagen (NTx) in urine by ELISA methods.

The mean daily urinary Na output decreased significantly (from 6.3 to $4.6 \mathrm{~g}$ salt/d) on the reduced-salt dietary regimen (see Table). While diastolic BP was unaffected by dietary salt regimen, systolic BP was significantly reduced (by $3 \mathrm{mmHg}$ on average) on the reducedsalt diet. This effect remained after adjustment in regression model for potential confounding effect of age, sex, weight, smoking and vitamin $\mathrm{D}$ status $(\beta=-6.04 ; P=0.001)$.

\begin{tabular}{lcrrrr}
\hline & \multicolumn{2}{c}{ Usual Na } & \multicolumn{2}{c}{ Reduced Na } & \multirow{2}{*}{$P$-value* } \\
\cline { 2 - 5 } Diet period & Mean & SD & Mean & SD & 0.0001 \\
Urinary Na (mmol/d) & 108 & 54 & 79 & 35 & 0.0001 \\
Systolic BP (mmHg) & 134 & 12 & 131 & 11 & 0.8 \\
Diastolic BP (mmHg) & 85 & 9 & 85 & 8 & \\
\hline
\end{tabular}

* $P$-value for treatment effect, there was no significant $(P>0.4)$ carry-over effects.

There was no significant effect of dietary salt regimen on the serum or urinary makers of bone turnover (data not shown).

In conclusion, consumption of a low-salt bread, unsalted spread and reduced-salt luncheon beef and turkey together with other pragmatic advise for limiting salt intake lead to a significant reduction in systolic BP in this group of adults with stage 1 hypertension, illustrating that a dietary approach to BP reduction is a sensible first step. There was no beneficial effect of reducing dietary salt on the rate of bone turnover.

Funded by the Irish Government Department of Agriculture, Food and Fisheries through the Food Institutional Research Measure.

1. UK Scientific Advisory Committee on Nutrition (2003) Salt and Health Report. http://www.food.gov.uk/multimedia/pdfs/sacnforumreport.pdf

2. Cashman K et al. (2003) In Nutritional Aspects of Bone Health, pp. 267-289 [New S and Bonjour P-J, editors], Cambridge.

3. Food Safety Authority of Ireland (2005) Salt and Health: Review of the Scientific Evidence and Recommendations for Public Policy in Ireland. Dublin: FSAI.

4. Lynch EJ et al. (2009) Food Res Int 7, 885-891. 